\title{
Subtleties of the clock retardation
}

\author{
D V Redžić \\ Faculty of Physics, University of Belgrade, PO Box 44, 11000 Beograd, Serbia \\ E-mail: redzic@ff.bg.ac.rs
}

\begin{abstract}
For a simple electromagnetic model of a clock introduced by Jefimenko (clock \# 1 in 1996 Am. J. Phys. 64 812), a change of the rate of the clock when it is set in uniform motion is calculated exactly, employing the correct equation of motion of a charged particle in the electromagnetic field and the universal boostability assumption. Thus, for the clock under consideration, a dynamical content of the clock retardation is demonstrated. Somewhat surprisingly, the analysis presented discloses that some familiar relativistic generalisations concerning the retardation of clocks have to be amended.
\end{abstract}

PACS numbers: 03.30.+p; 41.20.-q

\section{Introduction}

Consider a thin massive ring of radius $a$ at rest in the laboratory frame $S$, carrying a uniformly distributed charge $q_{1}$. Let the axis of the ring be the $x$ axis, with the origin at the centre of the ring. The electrostatic field of the ring on its axis is

$$
\boldsymbol{E}(x, 0,0)=\frac{\kappa q_{1} x \hat{\boldsymbol{x}}}{\left(a^{2}+x^{2}\right)^{3 / 2}},
$$

where $\kappa \equiv 1 / 4 \pi \epsilon_{0}$. A point charge $q_{2}$ of opposite sign $\left(q_{1} q_{2}<0\right)$, whose mass is $m$, is set at the point on the positive $x$ axis with $x=\mathcal{A}$. If the charge $q_{2}$ is released with zero initial velocity to move under the action of the electrostatic field of the ring, it will oscillate along the axis between the points $x= \pm \mathcal{A}$. The system 'charged ring and $q_{2}$ ' thus constitutes a primitive electromagnetic clock (clock \# 1 in [1]); denote the period of oscillations of $q_{2}$ for the clock at rest by $T_{0}$.

Assume now that the ring and the point charge are set in motion with constant velocity $\boldsymbol{v}_{0}=v_{0} \hat{\boldsymbol{x}}$ along the positive $x$ axis. What is the period of the clock in uniform motion?

The problem was posed by Jefimenko, who solved it by a direct calculation of the period in the laboratory frame, employing Heaviside's formulae for the electric and magnetic fields of a uniformly moving point charge and the longitudinal mass of $q_{2}$ [1]. The author found that the period of oscillations of $q_{2}$ for the moving clock is

$$
T_{M}=\frac{T_{0}}{\sqrt{1-v_{0}^{2} / c^{2}}} .
$$


where $c^{2} \equiv 1 / \epsilon_{0} \mu_{0} ; c$ is the speed of electromagnetic waves in vacuo and at the same time the speed of light in vacuo. Thus the clock 'consisting of the charged ring and the point charge runs slower when the clock is moving, and the rate of the moving clock is $\left(1-v_{0}^{2} / c^{2}\right)^{-1 / 2}$ times the rate of the same stationary clock', as predicted by Special Relativity. Since the conclusion was reached via a dynamical argument only, Jefimenko inferred that, for the clock under consideration, his calculations 'provide a dynamic cause-and-effect type explanation of time dilation' [1]. Moreover, pursuing this line of reasoning, he noted that 'if the slow rate of moving clocks can be indeed explained as a dynamic cause-and-effect phenomenon rather than as the kinematic effect enunciated by Einstein, then the slow rate of moving clocks cannot be interpreted as a proof of time dilation' [2].

Unfortunately, Jefimenko's argument involves a confusing assumption that the velocity $v_{0}$ of the moving clock is much larger than the maximum velocity of $q_{2}$ relative to the ring; moreover, the desired conclusion (2) is reached due to the additional approximation of small oscillations, i. e., $\mathcal{A} \ll a$. Thus, while Jefimenko correctly pointed out a dynamical content of the clock retardation, his approach masks some important aspects of the phenomenon, making some of his inferences fallacious.

In this paper, an exact dynamical analysis of Jefimenko's clock \# 1 is given. It is demonstrated that if the clock in motion is to be relativistically valid, i. e. to serve as an identical standard of time also for a co-moving inertial observer, analysis of its clockwork requires two frames, the lab frame $S$ and the rest frame of the clock $S^{\prime}$. Thus, contrary to Jefimenko's claim, one cannot avoid Einstein's principle approach to Special Relativity; for understanding of the clockwork, a blend of constructive 'dynamical' and principle approaches is needed. Moreover, the analysis presented reveals that for this 'longitudinal' relativistic clock, while equation (2) still applies, the clock retardation exhibits non-uniformity, which seems to be overlooked in the literature.

Namely, Einstein in 1905 inferred from the Lorentz transformation, for a (practically) point clock, that a clock travelling with velocity $v$, 'when viewed from the stationary system', runs slower by the factor $\left(1-v^{2} / c^{2}\right)^{-1 / 2}$ than the same clock at rest in the stationary system ([3], cf also [4]). Later, he generalised this statement to a clock with a second hand and even declared that 'every happening in a physical system slows down when the system is set into translational motion' [5]. (Møller paraphrased this by stating that 'any physical system which is moving relative to a system of inertia must have a slower course of development than the same system at rest' [6].) Thus, according to Einstein, not only moving clocks run slow but time itself is 'dilated' in moving systemst, 'but this slowing occurs only from the standpoint of a non-comoving coordinate system (observer)' [5]. However, our analysis of Jefimenko's 'longitudinal' clock demonstrates that Einstein's familiar generalisation about slowing down of processes in a moving physical system need to be amended. Generally, some happenings in a physical system speed up when the system is set into translational

$\dagger$ The somewhat misleading term 'time dilation' was probably introduced by Tolman [7]; incidentally, it appears that 'dilatation' would be the gramatically correct variant [8]. 
motion. Also, for a periodic process, in a physical system travelling with a velocity $v_{0}$ relative to a system of inertia, while equation (2) applies, generally it is not true that development of the process has a slower course by the factor $\left(1-v_{0}^{2} / c^{2}\right)^{-1 / 2}$ than in the same system at rest: the clock retardation may be non-uniform. In this paper, a dynamical content of the phenomenon is illustrated by a detailed examination of Jefimenko's clock \# 1 [1, 2].

\section{Direct calculation of the retardation of Jefimenko's clock}

Now we shall calculate exactly the period of Jefimenko's clock described in Introduction, when it is at rest in the lab frame $S$, and when it is in uniform motion with velocity $\boldsymbol{v}_{0}=v_{0} \hat{\boldsymbol{x}}$ along the $x$ axis (the axis of the ring) with respect to the lab. As is well known, in order to be relativistically valid, a clock must operate according to some Lorentz-covariant laws. This can be fulfilled for Jefimenko's clock, since its operation is based on Maxwell's equations (which can be made to be Lorentz-covariant (cf, e.g., [9], [10])) and the equation of motion of a charge $q^{*}$ in the electromagnetic field

$$
\frac{\mathrm{d}}{\mathrm{d} t}\left(\frac{m^{*} \boldsymbol{v}}{\sqrt{1-v^{2} / c^{2}}}\right)=q^{*} \boldsymbol{E}+q^{*} \boldsymbol{v} \times \boldsymbol{B},
$$

where $m^{*}$ is the mass of the charge, $\boldsymbol{v}$ is its instantaneous velocity, $\boldsymbol{E}$ is the electric field and $\boldsymbol{B}$ is the magnetic flux density; the time parameter $t$ in the $S$ frame is interpreted in the standard way, employing propagation of light signals in vacuo as the absolute time keeper, assuming that Einstein's clock synchronisation is a valid procedure [6]. Equation

(3) fits the experimental facts if the additional independent assumption that $m^{*}$ is timeindependent is introduced; with that assumption, equation (3) can be Lorentz-covariant too [1].

\subsection{Clock at rest}

In this subsection we discuss the clockwork of Jefimenko's clock at rest.

The correct equation of motion of the charge $q_{2}$ in an electric field is

$$
m \frac{\mathrm{d}}{\mathrm{d} t}\left(\frac{\boldsymbol{v}}{\sqrt{1-v^{2} / c^{2}}}\right)=q_{2} \boldsymbol{E},
$$

where the mass $m$ of $q_{2}$ is assumed to be time-independent. Equation (4) and identity

$$
\boldsymbol{v} \cdot \frac{\mathrm{d}}{\mathrm{d} t}\left(\frac{\boldsymbol{v}}{\sqrt{1-v^{2} / c^{2}}}\right) \equiv c^{2} \frac{d}{d t}\left(\frac{1}{\sqrt{1-v^{2} / c^{2}}}\right)
$$

imply that

$$
m c^{2} \frac{\mathrm{d}}{\mathrm{d} t}\left(\frac{1}{\sqrt{1-v^{2} / c^{2}}}\right)=q_{2} \boldsymbol{E} \cdot \boldsymbol{v} .
$$


Specifying to our problem, $\boldsymbol{E}$ is the electrostatic field of the ring on its axis given by equation (1), and $\boldsymbol{v}=v_{x} \hat{\boldsymbol{x}}$, since the motion is along the $x$ axis. Using equations (4), (6) and (1) one obtain $\ddagger$

$$
\frac{\mathrm{d} v_{x}}{\mathrm{~d} t}=-\frac{\kappa\left|q_{1} q_{2}\right|}{m}\left(1-\frac{v_{x}^{2}}{c^{2}}\right)^{3 / 2} \frac{x}{\left(a^{2}+x^{2}\right)^{3 / 2}},
$$

which can obviously recast into

$$
\frac{\mathrm{d} v_{x}}{\mathrm{~d} x} v_{x}=-\frac{\kappa\left|q_{1} q_{2}\right|}{m}\left(1-\frac{v_{x}^{2}}{c^{2}}\right)^{3 / 2} \frac{x}{\left(a^{2}+x^{2}\right)^{3 / 2}} .
$$

Separating variables and integrating, setting $v_{x}=0$ when $x=\mathcal{A}$ and solving for $v_{x}$ yields

$$
v_{x}=\frac{\mathrm{d} x}{\mathrm{~d} t}=\mp c\{\ldots\}^{1 / 2},
$$

where - and + sign corresponds to the motion of $q_{2}$ in the direction of decreasing $x$ and increasing $x$, respectively, and

$$
\{\ldots\} \equiv\left\{1-\left[1+\left(\kappa\left|q_{1} q_{2}\right| / m c^{2}\right)\left(1 / \sqrt{a^{2}+x^{2}}-1 / \sqrt{a^{2}+\mathcal{A}^{2}}\right)\right]^{-2}\right\} .
$$

Equation (9) implies that, for the oscillator at rest, passage of $q_{2}$ from $x$ to $x+\mathrm{d} x$ lasts time interval

$$
\mathrm{d} t=\mp(1 / c)\{\ldots\}^{-1 / 2} \mathrm{~d} x .
$$

Thus, the period $T_{0}$ of the oscillator at rest is given by $\S$

$$
T_{0}=\frac{2}{c} \int_{-\mathcal{A}}^{\mathcal{A}}\{\ldots\}^{-1 / 2} \mathrm{~d} x .
$$

\subsection{Clock in uniform motion}

Assume now that the same clock is set in uniform motion along the $x$ axis (the axis of the ring) with constant velocity $\boldsymbol{v}_{0}=v_{0} \hat{\boldsymbol{x}}$, so as to be relativistically valid, i. e. to serve as an identical standard of time also for a co-moving observer. The $x$ coordinate of the charge $q_{2}, x$, can be expressed as

$$
x=x_{c}+x_{r},
$$

$\ddagger$ The force $q_{2} \boldsymbol{E}$ is always parallel to the instantaneous velocity $\boldsymbol{v}$ of the charge $q_{2}$ so that equation (7) can be derived using the concept of 'longitudinal' mass, taking into account that the Lorentz force expression is a pure force ( $\mathrm{cf}[12$, 11]); I preferred not to employ here the potentially misleading concept of 'longitudinal' mass.

$\S$ Incidentally, in the case of small oscillations, i.e. when $\mathcal{A} \ll a$, from equation (12) one obtains that

$$
T_{0} \approx \frac{2}{c} \int_{-\mathcal{A}}^{\mathcal{A}}\left\{1-\left[1+\left(\mathcal{K} / 2 m c^{2}\right)\left(\mathcal{A}^{2}-x^{2}\right)\right]^{-2}\right\}^{-1 / 2} \mathrm{~d} x .
$$

where $\mathcal{K} \equiv \kappa\left|q_{1} q_{2}\right| / a^{3}$. Note that when $\mathcal{K} \mathcal{A}^{2} \ll m c^{2}$, from the last equation one obtains the familiar expression for the period of the simple harmonic oscillator, $T_{0}=2 \pi \sqrt{m / \mathcal{K}}$ (cf also [13], [14]). 
where $x_{c}$ is the $x$ coordinate of the centre of the ring, and $x_{r} \equiv x-x_{c}$ is the relative coordinate of $q_{2}$ with respect to the instantaneous position of the centre. Since $v_{x}=\mathrm{d} x / \mathrm{d} t$ and $v_{0}=\mathrm{d} x_{c} / \mathrm{d} t$, one has

$$
v_{x}=v_{0}+v_{x r}
$$

where $v_{x r}=\mathrm{d} x_{r} / \mathrm{d} t$ is the relative velocity of $q_{2}$ with respect to the centre of the ring, measured in the lab frame $S$.

The charge $q_{2}$ moves under the action of the electromagnetic field on the axis of the uniformly moving charged ring (the ring constitutes the framework of Jefimenko's clock). The electromagnetic field is calculated using the formulae for the $\boldsymbol{E}$ and $\boldsymbol{B}$ fields of a point charge $q$ moving with constant velocity $\boldsymbol{v}_{0}$, that were first obtained by Heaviside [15, 16] (the $\boldsymbol{B}$ field was rederived by J J Thomson [17], cf Jefimenko [18] and references therein), long before the advent of Special Relativity. The electric field of $q$ (radial but not spherically symmetrical) is given by

$$
\boldsymbol{E}(\boldsymbol{r}, t)=\frac{\kappa q \boldsymbol{r}}{r^{3}} \frac{1-v_{0}^{2} / c^{2}}{\left(1-v_{0}^{2} \sin ^{2} \theta / c^{2}\right)^{3 / 2}},
$$

where $\boldsymbol{r}$ is the position vector of a field point with respect to the instantaneous (at the same instant t) position of $q, \theta$ is the angle between $\boldsymbol{r}$ and the velocity $\boldsymbol{v}_{0}$, and $c^{2} \equiv 1 / \epsilon_{0} \mu_{0}$. (Recall that throughout the relativity paper [3], Einstein used the same symbol $(V)$ for the speed of light in vacuo and the speed of electromagnetic waves in vacuo $\left(V \equiv 1 / \sqrt{\epsilon_{0} \mu_{0}}\right.$ in SI system, not used by Einstein, he employed the HeavisideLorentz units), linking thus Special Relativity with Maxwell's theory (cf [19], p 197).) The magnetic flux density is

$$
\boldsymbol{B}(\boldsymbol{r}, t)=\epsilon_{0} \mu_{0} \boldsymbol{v}_{0} \times \boldsymbol{E}(\boldsymbol{r}, t) .
$$

A simple analysis employing formula (15) gives the following expression for the electric field on the axis of the moving charged ring

$$
\boldsymbol{E}_{M}=\frac{\kappa q_{1}\left(1-v_{0}^{2} / c^{2}\right) x_{r} \hat{\boldsymbol{x}}}{\left(a^{2}+x_{r}^{2}-a^{2} v_{0}^{2} / c^{2}\right)^{3 / 2}},
$$

as Jefimenko pointed out; the subscript $M$ serves as a reminder that the field is due to the moving ring-charge. The $\boldsymbol{B}$ field on the axis of the ring obviously vanishes. Using equations (4), (6) and (17) one obtains

$$
\frac{\mathrm{d} v_{x}}{\mathrm{~d} t}=-\frac{\kappa\left|q_{1} q_{2}\right|}{m}\left(1-\frac{v_{x}^{2}}{c^{2}}\right)^{3 / 2} \frac{\left(1-v_{0}^{2} / c^{2}\right) x_{r}}{\left(a^{2}+x_{r}^{2}-a^{2} v_{0}^{2} / c^{2}\right)^{3 / 2}} .
$$

Using equation (14), the last equation can obviously be recast into

$$
\frac{\mathrm{d} v_{x}\left(v_{x}-v_{0}\right)}{\left(1-v_{x}^{2} / c^{2}\right)^{3 / 2}}=-\frac{\kappa\left|q_{1} q_{2}\right|}{m} \frac{x_{r} \mathrm{~d} x_{r}\left(1-v_{0}^{2} / c^{2}\right)}{\left[a^{2}\left(1-v_{0}^{2} / c^{2}\right)+x_{r}^{2}\right]^{3 / 2}} .
$$

Now we have to specify our clock so as to be relativistically valid. As can be seen, this cannot be done without recourse to Einstein's principle approach to Special 
Relativity, i. e. without employing the Lorentz transformation and the universal boostability assumption (cf [14, 19]). A little reflexion reveals that one has to choose initial condition $v_{x}=v_{0}$ for $x_{r}=\mathcal{A} \sqrt{1-v_{0}^{2} / c^{2}}$. A glance at equation (18) shows that the charge $q_{2}$ will oscillate between the points $x_{r}= \pm \mathcal{A} \sqrt{1-v_{0}^{2} / c^{2}}$, where $v_{x}=v_{0}$.

Integration of equation (19), taking into account the initial condition, gives

$$
\frac{1-v_{0} v_{x} / c^{2}}{\sqrt{1-v_{x}^{2} / c^{2}}} \frac{1}{\sqrt{1-v_{0}^{2} / c^{2}}}=1+\gamma
$$

where

$$
\gamma \equiv \frac{\kappa\left|q_{1} q_{2}\right|}{m c^{2}}\left(\frac{1}{\sqrt{a^{2}+x_{r}^{2} /\left(1-v_{0}^{2} / c^{2}\right)}}-\frac{1}{\sqrt{a^{2}+\mathcal{A}^{2}}}\right) .
$$

Solving for $v_{x}$ and using equation (14), after a somewhat lengthy but in every step simple calculation, skipping details, one obtains for $v_{x r}$

$$
v_{x r}=\frac{\mathrm{d} x_{r}}{\mathrm{~d} t}=\mp \frac{c\left(1-v_{0}^{2} / c^{2}\right) \sqrt{1-1 /(1+\gamma)^{2}}}{1 \mp\left(v_{0} / c\right) \sqrt{1-1 /(1+\gamma)^{2}}},
$$

where - and + sign corresponds to the motion of $q_{2}$ in the direction of decreasing $x_{r}$ and increasing $x_{r}$, respectively. Equation (22) obviously implies that passage of $q_{2}$ from $x_{r}$ to $x_{r}+\mathrm{d} x_{r}$ lasts time interval

$$
\mathrm{d} t=\mp \frac{1 \mp\left(v_{0} / c\right) \sqrt{1-1 /(1+\gamma)^{2}}}{c\left(1-v_{0}^{2} / c^{2}\right) \sqrt{1-1 /(1+\gamma)^{2}}} \mathrm{~d} x_{r},
$$

Introducing

$$
x_{r}^{*}=\frac{x_{r}}{\sqrt{1-v_{0}^{2} / c^{2}}},
$$

and

$$
\gamma^{*}=\gamma=\frac{\kappa\left|q_{1} q_{2}\right|}{m c^{2}}\left(\frac{1}{\sqrt{a^{2}+x_{r}^{* 2}}}-\frac{1}{\sqrt{a^{2}+\mathcal{A}^{2}}}\right) .
$$

equation (23) can be recast into

$$
\mathrm{d} t=\mp \frac{\left[1 / \sqrt{1-1 /\left(1+\gamma^{*}\right)^{2}} \mp v_{0} / c\right]}{\sqrt{1-v_{0}^{2} / c^{2}} c} \mathrm{~d} x_{r}^{*},
$$

or, equivalently,

$$
\mathrm{d} t=\mp \frac{1}{\sqrt{1-v_{0}^{2} / c^{2}}} \frac{1}{c}\left[\left\{\ldots^{*}\right\}^{-1 / 2} \mp v_{0} / c\right] \mathrm{d} x_{r}^{*},
$$

where

$$
\left\{\ldots^{*}\right\} \equiv\left\{1-\left[1+\left(\kappa\left|q_{1} q_{2}\right| / m c^{2}\right)\left(1 / \sqrt{a^{2}+x_{r}^{* 2}}-1 / \sqrt{a^{2}+\mathcal{A}^{2}}\right)\right]^{-2}\right\} .
$$

Comparing equations (27) and (11), taking into account that $x_{r}^{*}$ runs from $\mathcal{A}$ to $-\mathcal{A}$ and vice versa, it follows that clock retardation is non-uniform in the case of Jefimenko's 
clock; simple slowing down by the factor $1 / \sqrt{1-v_{0}^{2} / c^{2}}$ is clearly violated in the 'life' of this 'longitudinal' clock. $\llbracket$ Particularly, using equation (27) one finds that travelling of $q_{2}$ downwards from $x_{r}=\mathcal{A} \sqrt{1-v_{0}^{2} / c^{2}}$ to $x_{r}=-\mathcal{A} \sqrt{1-v_{0}^{2} / c^{2}}$ lasts time interval

$$
(\Delta t)_{\text {down }}=-\frac{1}{\sqrt{1-v_{0}^{2} / c^{2}}} \frac{1}{c}\left[\int_{\mathcal{A}}^{-\mathcal{A}}\left\{\ldots^{*}\right\}^{-1 / 2} \mathrm{~d} x_{r}^{*}-\int_{\mathcal{A}}^{-\mathcal{A}} \frac{v_{0}}{c} \mathrm{~d} x_{r}^{*}\right],
$$

whereas the reverse travel upwards lasts

$$
(\Delta t)_{\mathrm{up}}=\frac{1}{\sqrt{1-v_{0}^{2} / c^{2}}} \frac{1}{c}\left[\int_{-\mathcal{A}}^{\mathcal{A}}\left\{\ldots^{*}\right\}^{-1 / 2} \mathrm{~d} x_{r}^{*}+\int_{-\mathcal{A}}^{\mathcal{A}} \frac{v_{0}}{c} \mathrm{~d} x_{r}^{*}\right] .
$$

Taking into account equation (12), these expressions can be recast into

$$
\begin{aligned}
& (\Delta t)_{\text {down }}=\frac{1}{\sqrt{1-v_{0}^{2} / c^{2}}}\left(\frac{T_{0}}{2}-\frac{v_{0}}{c^{2}} 2 \mathcal{A}\right), \\
& (\Delta t)_{\mathrm{up}}=\frac{1}{\sqrt{1-v_{0}^{2} / c^{2}}}\left(\frac{T_{0}}{2}+\frac{v_{0}}{c^{2}} 2 \mathcal{A}\right) .
\end{aligned}
$$

Consequently, for the period of Jefimenko's uniformly moving 'longitudinal' clock, one obtains

$$
T_{M}=(\Delta t)_{\mathrm{down}}+(\Delta t)_{\mathrm{up}}=\frac{T_{0}}{\sqrt{1-v_{0}^{2} / c^{2}}},
$$

as expected for a clock the clockwork of which is based on some Lorentz-covariant (or that can be made to be Lorentz-covariant, cf, e. g., [9, 10]) laws; the period of the clock in motion is by the factor $1 / \sqrt{1-v_{0}^{2} / c^{2}}$ greater than the period of the same clock at rest, all with respect to the lab frame $S$. However, the present analysis reveals a dynamical content of the phenomenon and its non-uniform character, which are hidden in the conventional 'kinematical' approach.

\section{Discussion}

The above analysis of Jefimenko's 'longitudinal' clock demonstrates that the clock retardation is basically of a dynamical origin, a consequence of velocity-dependence of the forces governing the operation of the clock. However, it also demonstrates that an exact one frame derivation of the clock retardation for this 'longitudinal' type of clock is not possible; in order to specify the clock so as to be relativistically valid (inter alia, to choose adequately the amplitude of oscillations of $q_{2}$ for the clock in motion), one has to recourse to Einstein's principle approach to Special Relativity Thus, perhaps somewhat surprisingly for adherents of a constructive dynamical approach to the theory,

\| This is in contradistinction to the case of a 'transverse' clock where the clock retardation is uniform. A dynamical content of the clock retardation in the case of a simple electromagnetic model of a 'transverse' relativistically valid clock was recently analysed exactly in [14.

I This is analogous to the case of a 'transverse' clock, where it seems that an exact one frame derivation of the clock retardation is impossible too ( $\mathrm{cf}[14]$ ). 
it appears that only a blend of constructive dynamical and principle approaches leads to a complete insight into phenomena.

Another outcome of our analysis of Jefimenko's clock is that some familiar relativistic generalisations concerning time need to be amended. Notably, declarations such as 'any physical system which is moving relative to a system of inertia must have a slower course of development than the same system at rest' [6], prove to be fallacious in the general case + However, Möller's statement is valid in the special case of a periodic complete process occurring in Jefimenko's clock. Indeed 'moving clocks run slow' from the standpoint of a non-comoving observer, but with addendum that within one period this slowing is generally non-uniform.

The power and precision of Einstein's principle approach to Special Relativity manifests itself in the universality of its results, regardless of the type of Lorentzcovariant mechanism responsible for a particular phenomenon. Therefore it is to be expected that some of our basic conclusions concerning the properties of relativistically valid clocks in uniform motion are reachable also via the principle approach. This is indeed so, as the following argument reveals.

Consider the standard Lorentz transformation

$$
t=\frac{t^{\prime}+v_{0} x^{\prime} / c^{2}}{\sqrt{1-v_{0}^{2} / c^{2}}}, \quad x=\frac{x^{\prime}+v_{0} t^{\prime}}{\sqrt{1-v_{0}^{2} / c^{2}}}, \quad y=y^{\prime}, \quad z=z^{\prime},
$$

where unprimed coordinates refer to the lab frame $S$ and primed coordinates refer to an inertial frame $S^{\prime}$ which is in standard configuration with $S$ ( $S^{\prime}$ is uniformly moving at speed $v_{0}$ along the common positive $x, x^{\prime}$-axes, and the $y$-and $z$-axis of $S$ are parallel to the $y^{\prime}$ - and $z^{\prime}$-axis of $S^{\prime}$ ).

Let $S^{\prime}$ be the rest frame of the framework of a relativistically valid clock. For Einstein's (practically) point clock, the first equation (34) and the condition $x^{\prime}=$ const imply the familiar result

$$
\mathrm{d} t=\frac{\mathrm{d} t^{\prime}}{\sqrt{1-v_{0}^{2} / c^{2}}} .
$$

Consider now a 'transverse' relativistically valid clock, i. e. the one the clockwork of which involves oscillations of a material point with $x^{\prime}=$ const, whereas $y^{\prime}$ and $z^{\prime}$ are variable (cf [14]). Clearly, equation (35) applies to this type of clock too. Taking into account the principle of (special) relativity, and the universal boostability assumption [19, 21], equation (35) can be given the following interpretation: any segment of 'life'

+ Möller's statement is clearly wrong in the case of the 'longitudinal' light-pulse clock (cf, e. g., [20, pp 105-9). Namely, a simple analysis reveals that the 'downwards' segment of life of the moving light clock (i. e., duration of the motion of light-pulse in the direction opposite to the direction of motion of the clock itself) lasts shorter than the corresponding segment of life of the same clock at rest, all with respect to the lab frame $S$, since

$$
\frac{l_{0} \sqrt{1-v_{0}^{2} / c^{2}}}{c+v_{0}}<\frac{l_{0}}{c},
$$

where $l_{0}$ is the rest length of the clock and $v_{0}$ is its velocity relative to $S$. 
of a 'transverse' clock that is moving uniformly with the velocity $v_{0}$ lasts longer by the factor $1 / \sqrt{1-v_{0}^{2} / c^{2}}$ than the same segment of 'life' of the same clock at rest in $S$, all with respect to the lab frame $S[14]$.

Finally, consider a 'longitudinal' relativistically valid clock, which involves oscillations of a material point with $x^{\prime} \neq$ const, and $y^{\prime}$ and $z^{\prime}$ need not be constant. In this case, the first equation (34) implies

$$
\mathrm{d} t=\frac{\mathrm{d} t^{\prime}+\left(v_{0} / c^{2}\right) \mathrm{d} x^{\prime}}{\sqrt{1-v_{0}^{2} / c^{2}}}=\frac{\mathrm{d} t^{\prime}+\left(v_{0} / c^{2}\right) v_{x}^{\prime} \mathrm{d} t^{\prime}}{\sqrt{1-v_{0}^{2} / c^{2}}} .
$$

where $v_{x}^{\prime}$ is the $x^{\prime}$ component of the instantaneous velocity of the material point in $S^{\prime}$. As can be seen, the first equation (36) is tantamount to equation (27), taking into account the universal boostability assumption. Obviously, equation (35) does not apply to this 'longitudinal' type of clock; the clock retardation now is non-uniform, contrary to a general consensus in the literature that equation (35) is universally valid. However, any clock involves by definition a periodic process; thus, integration of equation (36) over a period in $S^{\prime}$ yields

$$
T=\frac{T^{\prime}}{\sqrt{1-v_{0}^{2} / c^{2}}},
$$

where $T$ and $T^{\prime}$ are the periods of the clock as observed in $S$ and $S^{\prime}$, respectively, since $x^{\prime}$ takes its initial value after the period $T^{\prime}$. As can be seen, equation (37) can be given the same meaning as equation $(33) *$

On the other hand, in the case of an aperiodic process involving a point particle, no inference can be deduced from equation (36), except that it is not generally true that 'every happening in a physical system slows down when the system is set into translational motion [in a rest properties-preserving way]' [5]. This implies that particular instances of 'time retardation' must be carefully considered.

Note that the universality and elegance of the above 'kinematical' deductions concerning clocks in motion may be deceptive. Namely, one should keep in mind that any clock retardation involves a complex dynamical process, which is completely masked in the conventional 'kinematical' derivation.

One last point. Basic inferences concerning slowing down of moving clocks from the standpoint of a non-comoving observer are deduced from Einstein's two principles of Special Relativity, aided with the universal boostability assumption. The present discussion reveals that for subtler insights, dynamical considerations are needed, as expected. Moreover, the dynamical approach indicates where to look within the principle approach. On the other hand, the principle approach anticipates simplicity and universality behind an intricate dynamical mechanism; for instance, one knows

* But, we derived equation (33) for Jefimenko's clock, which is strictly 'longitudinal' $\left(y^{\prime}=z^{\prime}=\right.$ 0), whereas the 'kinematical' approach yields equation (37) for more general 'longitudinal' clocks. Incidentally, any real clock involves damping; thus, Jefimenko's clock represents an ideal clock since the radiation reaction force was neglected in its clockwork. 
in advance that $T_{M}$ depends on dynamical parameters only through dependence of $T_{0}$ on those parameters. Thus one can evade cumbersome dynamical calculation for a physical system in uniform motion with respect to the lab frame by combining much simpler dynamical calculation in the rest frame of the system and the transformation laws of relevant quantities known from the principle approach, as Einstein noted long ago for 'all problems in the optics of moving bodies' [3].

In his excellent book Special Relativity, French warned to beware of 'glib statements involving relativity theory' 20]. This paper, hopefully, represents such a warning, illustrating ever present danger of unjustified generalisations.

\section{Acknowledgments}

My work is supported by the Ministry of Science and Education of the Republic of Serbia, project No. 171028.

\section{References}

[1] Jefimenko O D 1996 Direct calculation of time dilation Am. J. Phys. 64 812-4

[2] Jefimenko O D 1998 On the experimental proofs of relativistic length contraction and time dilation Z. Naturforsch. 53a $977-82$

[3] Einstein A 1905 Zur Elektrodynamik bewegter Körper Ann. Phys., Lpz. 17 891-921

[4] Einstein A 1916 Die Grundlage der allgemeinen Relativitätstheorie Ann. Phys., Lpz. 49 769-822

[5] Einstein A 1925 Die Relativitätstheorie, in Kultur der Gegenwart: Physik 2nd ed (Leipzig: Teubner) pp 783-97

[6] Møller C 1972 The Theory of Relativity 2nd edn (Oxford: Clarendon)

[7] Tolman R C 1934 Relativity, Thermodynamics, and Cosmology (Oxford: Oxford U. P.) pp 22-5

[8] Sears F W 1964 Time dila(tat)tion Am. J. Phys. 32570

[9] Rosser W G V 1964 An Introduction to the Theory of Relativity (London: Butterworths)

[10] Redžić D V 2014 Force exerted by a moving electric current on a stationary or co-moving charge: Maxwell's theory versus relativistic electrodynamics Eur. J. Phys. 35045011

[11] Redžić D V, Davidović D M and Redžić M D 2011 Derivations of relativistic force transformation equations J. Electro. Waves Appl. 25 1146-55

[12] Rindler W 1991 Introduction to Special Relativity 2nd ed (Oxford: Clarendon)

[13] Møller C 1955 Old problems in the general theory of relativity viewed from a new angle Mat. Fys. Medd. Dan. Vid. Selsk. 30 issue 10

[14] Redžić D V 2015 Direct calculation of length contraction and clock retardation Serbian Astronomical Journal 190 in press

[15] Heaviside O 1888 The electro-magnetic effects of a moving charge Electrician 22 147-8

[16] Heaviside O 1889 On the electromagnetic effects due to the motion of electrification through a dielectric Philos. Mag. 27 324-39

[17] Thomson J J 1889 On the magnetic effects produced by motion in the electric field Philos. Mag. 28 1-14

[18] Jefimenko O D 1994 Direct calculation of the electric and magnetic fields of an electric point charge moving with constant velocity Am. J. Phys. 62 79-85

[19] Redžić D V 2008 Towards disentangling the meaning of relativistic length contraction Eur. J. Phys. 29 191-201

[20] French A P 1968 Special Relativity (London: Nelson)

[21] Redžić D V 2014 Relativistic length agony continued Serbian Astronomical Journal 188 55-65 